\title{
Uma avaliação da eficiência energética de uma Rede de Sensores Sem Fios em relação ao posicionamento do nó sink
}

\author{
Melissa Bonfim Alcantud ${ }^{1}$, César Alberto da Silva ${ }^{1,2}$, Andrea Padovan Jubileu ${ }^{1}$, \\ Linnyer Beatryz Ruiz ${ }^{2,3}$
}

${ }^{1}$ Departamento de Informática - Instituto Federal de São Paulo (IFSP)

CEP 19470-000 - Presidente Epitácio, SP - Brasil

${ }^{2}$ Programa de Pós-Graduação em Engenharia Elétrica

Universidade Federal de Minas Gerais (UFMG) - Belo Horizonte, MG - Brasil

${ }^{3}$ Programa de Pós-Graduação em Ciências da Computação

Universidade Estadual de Maringá (UEM) - Maringá, PR - Brasil

\{melissa.alcantud, casilva91, andreapjubileu,linnyer\}@gmail.com

\begin{abstract}
The research on the use of wireless technologies in several areas propelled this study regarding the energy efficiency of Wireless Sensor Networks (WSN), considering the variation of the number of sensor nodes, the time of data collection of these nodes and the positioning of the sink node. For this study, the Castalia simulator was used as an instrument to analyze the behavior of an WSN in a real environment, reducing cost and time.
\end{abstract}

Resumo. A pesquisa em relação ao uso de tecnologias de conexão sem fio em diversas áreas propulsionou esse estudo referente à eficiência energética de Redes de Sensores sem Fio (RSSF), considerando a variação de quantidade de nós sensores, o tempo de coleta de dados desses nós e o posicionamento do nó sink. Para a realização desse estudo foi utilizado o simulador Castalia como instrumento de análise do comportamento de uma RSSF em um ambiente real, reduzindo custo e tempo.

\section{Introdução}

As Redes de Sensores Sem Fio (RSSFs) possuem uma ampla possibilidade de serem utilizadas em diferentes áreas, tais como agricultura, militar, ambiental, médica, entre outras. Podem ser implantadas para monitorar aspectos do mundo físico por meio dos nós sensores, que são pequenos computadores acoplados com sensores de temperatura, vibração e outros [Tanembaum e Wetherrall 2011].

A quantidade de energia consumida pelos nós sensores são quesitos relevantes na implantação de uma aplicação, visto que a vida útil das RSSFs pode estar relacionada à capacidade de hardware, armazenamento e recarga de energia [Ruiz et. al 2011] e [Stattner et. al 2012]. Para encontrar um modelo ideal de configuração, de acordo com aplicação desejada, faz-se o uso de ferramentas que realizam simulações de ambientes reais utilizando os dados coletados de uma RSSF. Uma vez realizada a simulação com os parâmetros adotados, esses simuladores geram um pacote de arquivos contendo dados que retratam todos os eventos que ocorreram ao longo da simulação na rede. Os 
dados coletados estão aptos a serem analisados estatisticamente, a fim de serem comparados para a escolha/definição da melhor configuração da rede. Entre as várias ferramentas de simulações, as mais conhecidas para redes de sensores sem fio são: Network Simulator 2 (NS-2), TraceGraph, MannaSim e Castalia. Neste trabalho foi utilizado o Castalia, um simulador sem fio derivado do simulador OMNeT++.

Este trabalho compreende a simulação de uma RSSF variando a quantidade de nós sensores, o posicionamento do nó sink e o tempo de coleta de dados. Trata-se do resultado de um projeto de iniciação científica realizado por uma aluna do curso superior de Tecnologia em Análise e Desenvolvimento de Sistemas do IFSP - Câmpus Presidente Epitácio.

Neste artigo são apresentados os trabalhos relacionados ao estudo realizado (seção 2), as simulações e resultados obtidos (seção 3) e, por fim, as conclusões (seção 4) e referências de embasamento teórico.

\section{Trabalhos Relacionados}

Existem diversos trabalhos na literatura que propõem vários protocolos para reduzir o consumo de energia em RSSF. Kumar [2014] apresenta um estudo com uso de protocolos baseados em cluster. O autor propõe dois protocolos para uso em RSSF heterôgena: Single-hop Energy-Efficient Clustering Protocol (S-EECP) e Multi-hop Energy-Efficient Clustering Protocol (M-EECP). A eleição dos cluster heads, em ambos os protocolos, é realizada por uma probabilidade baseada na relação entre a energia residual de cada nó e a energia média da rede. Dessa forma, os nós com maior quantidade de energia têm mais chances de serem eleitos. A diferença entre esses protocolos é simplesmente que o protocolo S-EECP é utilizado para comunicação single-hop e o protocolo M-EECP é usado para comunicação multi-hop. Dois experimentos foram realizados utilizando o simulador NS-2. No primeiro experimento foram definidos 100 nós sensores distribuídos uniformemente em uma área de $100 \mathrm{x}$ $100 \mathrm{~m}^{2}$. No segundo experimento foram definidos 200 nós sensores distribuídos uniformemente em uma área de 200 x $200 \mathrm{~m}^{2}$. Os resultados dos experimentos foram comparados com os protocolos EECT [Xin et. al 2008] e EEHC [Kumar et. al 2009]. Os protocolos S-EECP e M-EECP foram superiores em relação ao tempo de vida da rede e mantiveram o consumo de energia balanceado entre os cluster heads.

Diversas outras abordagens são utilizadas na implementação de um protocolo de roteamento. Mondal et. al (2016) utilizam fuzzy logic para eleição do cluster head, Gupta et. al (2016) utilizam a técnica Honey Bee Optimization para selecionar o próximo nó na transmissão dos dados e Bhatti e Kaur (2017) propõem um algoritmo de roteamento com sink móvel baseado em grid virtual.

O estudo em questão, apresentado neste artigo, diferencia-se dos encontrados na literatura por fazer uma análise do consumo de energia da rede em relação a quantidade de nós sensores, posicionamento do nó sink e do tempo de coleta dos nós sensores.

\section{Simulação e Resultados}

Para realizar as simulações do presente estudo foi utilizado o simulador Castalia. No caso, os nós sensores tem como finalidade medir a temperatura e umidade de uma área 
com 4 hectares (40.000 metros quadrados), o que corresponde a uma área de 200 metros de comprimento por 200 metros de largura. Na referida área, os nós sensores da rede são distribuídos em forma de grid, ou seja, de maneira regular sobre a área monitorada, e com mesma capacidade de hardware.

Foram simulados quatro cenários com mobilidade estacionária e com quantidade de nós de 25, 36, 49 e 81. Para cada cenário foram definidos dois tempos de sensoriamento para os nós sensores: 10 e 30 segundos. Em ambos os casos, o posicionamento do nó responsável em centralizar o recebimento de dados (nó sink) foi avaliado no centro e em um canto da área, sendo que as simulações para cada cenário foram repetidas 33 vezes. As Tabelas 1 e 2 apresentam os resultados das simulações em relação ao tempo de coleta de 10 e 30 segundos, respectivamente.

Tabela 1: Dados das redes simuladas com envio de um pacote a cada 10 segundos.

\begin{tabular}{cccccccc}
\hline & Qtd.de nós Nó sink & $\begin{array}{c}\text { Pacotes } \\
\text { Recebidos } \\
\text { (nó sink) }\end{array}$ & $\begin{array}{c}\text { Pacotes } \\
\text { Enviados }\end{array}$ & $\begin{array}{c}\text { Perda de } \\
\text { Pacotes } \\
\text { Enviados }\end{array}$ & $\begin{array}{c}\text { Energia } \\
\text { Consumida } \\
\text { (Joules) }\end{array}$ & $\begin{array}{c}\text { Energia } \\
\text { Consumida } \\
\text { /Pacotes } \\
\text { recebidos }\end{array}$ \\
\hline conf_6x6 & 36 & centro & 50,377 & 541,379 & 296,285 & 18,018 & 0,357 \\
conf_6x6 & 36 & canto & 26,083 & 438,883 & 195,816 & 17,605 & 0,674 \\
conf_7x7 & 49 & centro & 64,146 & 699,404 & 420,871 & 19,117 & 0,298 \\
conf_7x7 & 49 & canto & 35,488 & 736,022 & 460,252 & 19,179 & 0,540 \\
conf_8x8 & 81 & centro & 72,253 & 773,474 & 480,615 & 19,929 & 0,276 \\
conf_8x8 & 81 & canto & 45,922 & 921,17 & 629,839 & 20,28 & 0,442 \\
\hline
\end{tabular}

Tabela 2: Dados das redes simuladas com envio de um pacote a cada 30 segundos.

\begin{tabular}{lccccccc}
\hline & Qtd.de nós & Nó sink & $\begin{array}{c}\text { Pacotes } \\
\text { Recebidos } \\
\text { (nó sink) }\end{array}$ & $\begin{array}{c}\text { Pacotes } \\
\text { Enviados }\end{array}$ & $\begin{array}{c}\text { Perda de } \\
\text { Pacotes } \\
\text { Enviados }\end{array}$ & $\begin{array}{c}\text { Energia } \\
\text { Consumida } \\
\text { (Joules) }\end{array}$ & $\begin{array}{c}\text { Energia } \\
\text { Consumida / } \\
\text { Pacotes } \\
\text { recebidos }\end{array}$ \\
\hline conf_6x6 & 36 & centro & 19,729 & 361,089 & 105,002 & 17,538 & 0,888 \\
conf_6x6 & 36 & canto & 10,752 & 327,96 & 75,707 & 17,318 & 1,610 \\
conf_7x7 & 49 & centro & 25,869 & 439,683 & 153,764 & 18,315 & 0,708 \\
conf_7x7 & 49 & canto & 13,766 & 452,039 & 169,211 & 18,369 & 1,334 \\
conf_8x8 & 81 & centro & 30,193 & 480,153 & 184,732 & 18,868 & 0,625 \\
conf_8x8 & 81 & canto & 19,512 & 541,179 & 246,541 & 19,015 & 0,975 \\
\hline
\end{tabular}

Pode-se observar que, mesmo variando a quantidade de nós e o tempo de coleta, o posicionamento do nó sink no centro obteve melhor resultado, em todos os cenários, em relação à energia consumida pela quantidade de pacotes recebidos.

\section{Conclusões}

Após as várias simulações com diferentes configurações de redes, chegou-se à conclusão que a localização do nó sink está diretamente relacionada ao consumo de energia em uma RSSF. 
A localização do nó sink deve visar à diminuição da distância máxima entre ele e os outros nós sensores da rede, minimizando o problema de encaminhamento de dados por nós sensores intermediários para os nós sensores que não possuem capacidade de comunicação direta com o nó sink.

A quantidade de nós sensores da rede também está diretamente relacionada ao consumo de energia, pois um número maior de nós sensores causa aumento de dados trafegando pela rede, inclusive de dados de controle para descoberta de novas rotas.

\section{Referências}

Bhatti, R. e Kaur, G. (2017). Virtual Grid based energy efficient mobile sink routing algorithm for WSN, In: 2017 11th International Conference on Intelligent Systems and Control (ISCO), Coimbatore, pp. 30-33.

Gupta, A., Thakur, A., Saini, H. S., Kumar, R. e Kumar, N. (2016). H-IECBR: HBO based-Improved Energy Efficient Chain Based Routing protocol in WSN, In: 2016 IEEE 1st International Conference on Power Electronics, Intelligent Control and Energy Systems (ICPEICES), Delhi, pp. 1-4.

Kumar, D. (2014). Performance analysis of energy efficient clustering protocols for maximising lifetime of wireless sensor networks, in IET Wireless Sensor Systems, vol. 4, no. 1, pp. 9-16, March 2014.

Kumar, D., Aseri, T.C., e Patel, R.B. (2009). EEHC: energy efficient heterogeneous clustered scheme for wireless sensor networks, In: Elsevier Comput. Commun, 32, (4), pp. 662-667.

Mondal, S., Dutta, P., Ghosh, S. e Biswas, U. (2016). Energy efficient rough fuzzy set based clustering and cluster head selection for WSN, In: 2016 2nd International Conference on Next Generation Computing Technologies (NGCT), Dehradun, India, pp. 439-444.

Pantazis, N. A., Nikolidakis, S. A. e Vergados, D. D. (2013). Energy-Efficient Routing Protocols in Wireless Sensor Networks: A Survey, In: IEEE Communications Surveys \& Tutorials, vol. 15, no. 2, pp. 551-591.

Ruiz, L. B., Loureiro, A. A. F., Silva, F. A., Silva, T. R. M. B., Pozza, R. S., Costa, J. C. C., Gonçalves, P. C. e Silva, C. A. (2011). Engenharia de computação invisível. In: DE SOUZA, A. F.; MEIRA JR., W. (Org.), Atualizações em Informática 2011, capítulo 8. PUC-Rio.

Stattner, E., Vidot, N., Hunel, P. e Collard, M. (2012). Wireless sensor network for habitat monitoring: A counting heuristic. In: 2012 IEEE 37th Conference on Local Computer Networks Workshops (LCN Workshops), p. 753-760.

Tanenbaum, A. S e Wetherall, D. (2011). Redes de computadores. Tradução: Daniel Vieira. $5^{\text {a }}$ ed. São Paulo: Person.

Xin, G., Yong, X. W. e Fang, L. (2008). "An energy-efficient clustering technique for wireless sensor networks". Proc. Int. Conf. IEEE Networking, Architecture, and Storage, pp. 248-252. 